\title{
A NOTE ON DISJOINTNESS PRESERVING OPERATORS
}

\author{
B. DE PAGTER
}

ABSTRACT. In this paper we present some results concerning the automatic order boundedness of disjointness preserving operators on Riesz spaces (vector lattices).

Let $L$ and $M$ be Archimedean Riesz spaces. The linear mapping $T$ from $L$ into $M$ is called disjointness preserving if $T f \perp T g$ whenever $f \perp g$ in $L$. Observe that a positive linear mapping $T$ is disjointness preserving iff $T$ is a Riesz homomorphism.

The purpose of this note is to prove a basic result (Theorem 2) concerning disjointness preserving operators, which has many applications to the automatic order boundedness problem for disjointness preserving operators. First of all it provides a short and simple proof of a recent result of Yu. A. Abramovich [1] to the effect that a disjointness preserving operator $T$ with the additional property that $\inf _{n}\left(\left|T f_{n}\right|+\left|T g_{n}\right|\right)=0$ in $M$ whenever $f_{n}, g_{n} \rightarrow 0$ (r.u.) in $L$, is order bounded. We note that a similar result for band preserving operators on Archimedean Riesz spaces was proved by S. J. Bernau [6] (recall that the linear operator $T$ from $L$ into itself is called band preserving if $f \perp g$ implies that $T f \perp g$ ).

Furthermore, Theorem 2 has as an immediate corollary that any order bounded disjointness preserving operator $T$ can be written as the difference of two Riesz homomorphisms, a result due to M. Meyer [10].

Finally we shall use Theorem 2 to show that any disjointness preserving operator from $L$ into $M$ is order bounded on some order dense ideal in $L$, whenever $L$ is uniformly complete and $M$ satisfies some additional conditions (e.g. if $M$ is a normed Riesz space). In particular, it follows from a combination of Proposition 6 and Theorem 8 that any band preserving operator on a Banach lattice is order bounded (and hence norm bounded), which is a result of Yu. Abramovich, A. I. Veksler and A. V. Koldunov [2].

For terminology used and properties of Riesz spaces not explained or proved in this paper, we refer to [4 or 9]. We start with a lemma.

LEMMA 1. Let $L$ be an Archimedean Riesz space and let $n$ be a fixed natural number. If $0 \leqslant u \leqslant e$ in $L$, then there exist $0 \leqslant p_{k} \in L(k=0,1, \ldots, 2 n)$ and $0 \leqslant v, w \in L$ such that

(i) $\sum_{k=0}^{2 n} p_{k}=u$,

Received by the editors March 10, 1983 and, in revised form, June 13, 1983.

1980 Mathematics Subject Classification. Primary 47B55; Secondary 06F20.

Key words and phrases. Disjointness preserving operator, automatic order boundedness, orthomorphism.

(C)1984 American Mathematical Society $0002-9939 / 84 \$ 1.00+\$ .25$ per page 
(ii) $|v-u| \leqslant e / n$ and $|w-u| \leqslant e / n$,

(iii) $v-k e / n \perp p_{2 k}$ for $k=0,1, \ldots, n$ and $w-(2 k-1) e / 2 n \perp p_{2 k-1}$ for $k=$ $1, \ldots, n$.

Proof. For $k=1,2, \ldots, 2 n$ put $y_{k}=[4 n u-(2 k-1) e]^{+} \wedge e$. Define

$$
v=\frac{1}{n} \sum_{k=1}^{n} y_{2 k}, \quad w=\frac{1}{2 n} y_{1}+\frac{1}{n} \sum_{k=2}^{n} y_{2 k-1},
$$

and $p_{k}=[4 n u-2 k e]^{+} \wedge u-[4 n u-2(k+1) e]^{+} \wedge u(k=0,1, \ldots, 2 n)$. We shall show that $\left\{p_{k}: k=0,1, \ldots, 2 n\right\}, v$ and $w$ fulfill the requirements. First of all, since $p_{0}=u-(4 n u-2 e)^{+} \wedge u$ and $p_{2 n}=0$, it is clear that (i) holds.

In order to prove (ii), observe that

$$
u=\sum_{k=1}^{n}\left[u-\left(\frac{k-1}{n}\right) e\right]^{+} \wedge\left(\frac{1}{n} e\right)
$$

and so

$$
|v-u| \leqslant \sum_{k=1}^{n}\left|\frac{1}{n} y_{2 k}-\left[u-\left(\frac{k-1}{n}\right) e\right]^{+} \wedge\left(\frac{1}{n} e\right)\right| .
$$

Since each term on the right-hand side is majorized by $e / n$, it is sufficient to show that these terms are mutually disjoint. Take $1 \leqslant k<l \leqslant n$. Since

$$
y_{2 l} \leqslant 4 n[u-((l-1) / n) e]^{+},
$$

it is sufficient to show that

$$
\frac{1}{n} y_{2 k}-\left[u-\left(\frac{k-1}{n}\right) e\right]^{+} \wedge\left(\frac{1}{n} e\right) \perp\left[u-\left(\frac{l-1}{n}\right) e\right]^{+},
$$

and hence we have to prove that

$$
[4 n u-(4 k-1) e]^{+} \wedge e-[n u-(k-1) e]^{+} \wedge e \perp(n u-k e)^{+} .
$$

Note that for $f, g \in L$ we have $|f \wedge e-g \wedge e| \leqslant(e-f)^{+} \vee(e-g)^{+},\left(e-f^{+}\right)^{+}$ $=(e-f)^{+} \wedge e$. Now

$$
\left(e-[4 n u-(4 k-1) e]^{+}\right)^{+}=(4 k e-4 n u)^{+} \wedge e \perp(n u-k e)^{+},
$$

and similarly

$$
\left(e-[n u-(k-1) e]^{+}\right)^{+}=(k e-n u)^{+} \wedge e \perp(n u-k e)^{+} .
$$

Hence the terms in the sum (1) are mutually disjoint and therefore $|v-u| \leqslant e / n$. The proof that $|w-u| \leqslant e / n$ follows the same lines.

In order to prove (iii) we first show that $e-y_{l} \perp p_{k}$ for $l \leqslant k$ and that $y_{l} \perp p_{k}$ for $l \geqslant k+2$. Suppose that $l \leqslant k$, then

$$
\begin{aligned}
e-y_{l} & =e-[4 n u-(2 l-1) e]^{+} \wedge e=\left(e-[4 n u-(2 l-1) e]^{+}\right)^{+} \\
& =(2 l e-4 n u)^{+} \wedge e \leqslant(2 k e-4 n u)^{+} \perp(4 n u-2 k e)^{+} \wedge u,
\end{aligned}
$$


and hence $e-y_{l} \perp p_{k}$. Now suppose that $l \geqslant k+2$, then

$$
\begin{aligned}
p_{k} & \leqslant u-[4 n u-2(k+1)]^{+} \wedge u=\left(u-[4 n u-2(k+1) e]^{+}\right)^{+} \\
& =[2(k+1) e-(4 n-1) u]^{+} \wedge u \leqslant[(2 l-2) e-(4 n-1) u]^{+} \\
& \leqslant[(2 l-1) e-4 n u]^{+} \perp[4 n u-(2 l-1) e]^{+},
\end{aligned}
$$

and hence $p_{k} \perp y_{l}$. Now

$$
v-\frac{k}{n} e=\frac{1}{n} \sum_{l=1}^{k}\left(y_{2 l}-e\right)+\frac{1}{n} \sum_{l=k+1}^{n} y_{2 l},
$$

and by the above $y_{2 l}-e \perp p_{2 k}(1 \leqslant l \leqslant k)$ and $y_{2 l} \perp p_{2 k}(k+1 \leqslant l \leqslant n)$. Hence $v-k e / n \perp p_{2 k}$ for $k=0,1, \ldots, n$. In like manner it is shown that $w-$ $(2 k-1) e / 2 n \perp p_{2 k-1}(k=1,2, \ldots, n)$.

THEOREM 2. Let $L$ and $M$ be Archimedean Riesz spaces and $T$ a disjointness preserving operator from $L$ into $M$. If $0 \leqslant u \leqslant e$ in $L$, then there exist two sequences $\left\{f_{n}\right\}_{n=1}^{\infty}$ and $\left\{g_{n}\right\}_{n=1}^{\infty}$, contained in the ideal generated by $e$ in $L$, such that $f_{n}, g_{n} \rightarrow 0$ (e-uniformly) and $(|T u|-|T e|)^{+} \leqslant\left|T f_{n}\right|+\left|T g_{n}\right|$ for all $n$.

Proof. Take $0 \leqslant u \leqslant e$ in $L$. By the above lemma there exist for each $n=1,2, \ldots$ elements $0 \leqslant p_{n k} \in L(k=0,1, \ldots, 2 n)$ and $0 \leqslant v_{n}, w_{n} \in L$ such that $v_{n}$ and $w_{n}$ are contained in the ideal generated by $e$, and

(i) $\sum_{k=0}^{2 n} p_{n k}=u$,

(ii) $\left|v_{n}-u\right| \leqslant e / n,\left|w_{n}-u\right| \leqslant e / n$,

(iii) $v_{n}-k e / n \perp p_{n, 2 k}(k=0,1, \ldots, n)$ and $w_{n}-(2 k-1) e / 2 n \perp p_{n, 2 k-1}(k=$ $1,2, \ldots, n)$.

Since $T$ is disjointness preserving, it follows from (iii) that

$$
T v_{n}-\frac{k}{n} T e \perp T p_{n, 2 k} \quad \text { and } \quad T w_{n}-\frac{2 k-1}{2 n} T e \perp T p_{n, 2 k-1} \quad(0 \leqslant k \leqslant n) .
$$

Furthermore, $\left(\left|T v_{n}\right|-|T e|\right)^{+} \leqslant\left|T v_{n}-k T e / n\right|$ and $\left(\left|T w_{n}\right|-|T e|\right)^{+} \leqslant \mid T w_{n}-$ $(2 k-1) T e / 2 n \mid$, which implies that $\left(\left|T v_{n}\right|-|T e|\right)^{+} \wedge\left(\left|T w_{n}\right|-|T e|\right)^{+} \perp T p_{n k}$, i.e., that

$$
\left(\left|T v_{n}\right| \wedge\left|T w_{n}\right|-|T e|\right)^{+} \wedge\left|T p_{n k}\right|=0 \quad \text { for all } k=0,1, \ldots, 2 n \text {. }
$$

Now it follows from (i) that $\left(\left|T v_{n}\right| \wedge\left|T w_{n}\right|-|T e|\right)^{+} \wedge|T u|=0$.

For all $n=1,2, \ldots$ we now have

$$
(|T u|-|T e|)^{+} \leqslant\left|T\left(u-v_{n}\right)\right|+\left(\left|T v_{n}\right|-|T e|\right)^{+}
$$

and

$$
(|T u|-|T e|)^{+} \leqslant\left|T\left(u-w_{n}\right)\right|+\left(\left|T w_{n}\right|-|T e|\right)^{+}
$$

hence

$$
\begin{aligned}
(|T u|-|T e|)^{+} \leqslant & \left\{\left|T\left(u-v_{n}\right)\right|+\left(\left|T v_{n}\right|-|T e|\right)^{+}\right\} \\
& \wedge\left\{\left|T\left(u-w_{n}\right)\right|+\left(\left|T w_{n}\right|-|T e|\right)^{+}\right\} \\
\leqslant & \left|T\left(u-v_{n}\right)\right|+\left|T\left(u-w_{n}\right)\right|+\left(\left|T v_{n}\right| \wedge\left|T w_{n}\right|-|T e|\right)^{+}
\end{aligned}
$$


Since $(|T u|-|T e|)^{+} \leqslant|T u|$, this implies that $(|T u|-|T e|)^{+} \leqslant\left|T\left(u-v_{n}\right)\right|+$ $\left|T\left(u-w_{n}\right)\right|$, and so we can take $f_{n}=u-v_{n}$ and $g_{n}=u-w_{n}$ for $n=1,2, \ldots$

The next corollary includes Abramovich's results [1, Proposition B].

Corollary 3. Let $L$ and $M$ be Archimedean Riesz spaces and $T$ a disjointness preserving operator from $L$ into $M$. If $T$ has the property that $\inf _{n}\left(\left|T f_{n}\right|+\left|T g_{n}\right|\right)=0$ whenever $f_{n}, g_{n} \rightarrow 0$ (r.u.) in $L$, then $0 \leqslant u \leqslant e$ in L implies that $|T u| \leqslant|T e|$ (and hence $T$ is order bounded).

COROllaRY 4 (SEE [10 AND 11]). Let L and $M$ be Archimedean Riesz spaces and let $T$ be an order bounded disjointness preserving operator from $L$ into $M$. Then $T=T^{+}$ $-T^{-}$, where $T^{+}$and $T^{-}$are Riesz homomorphisms and $T^{+} u=(T u)^{+}, T^{-} u=(T u)^{-}$ for all $0 \leqslant u \in L$.

Proof. Let $\hat{M}$ be the Dedekind completion of $M$ and consider $T$ as an operator from $L$ into $\hat{M}$. Since $T$ is order bounded, the absolute value $|T|$ exists as mapping from $L$ into $\hat{M}$. For each $0 \leqslant u \in L$ we have $|T|(u)=\sup \{|T f|:|f| \leqslant u\}$. Since $T$ is order bounded it is clear that the conditions of Corollary 3 are fulfilled, and so $|T f|=|T| f|| \leqslant|T u|$ for all $f \in L$ with $|f| \leqslant u$. Hence $|T|(u)=|T u|$, which shows that $|T|$ maps $L$ into $M$. Moreover, it follows from $T^{+}=\frac{1}{2}(|T|+T)$ and $T^{-}=\frac{1}{2}(|T|-T)$ that $T^{+} u=(T u)^{+}$and $T^{-} u=(T u)^{-}$for all $0 \leqslant u \in L$. Finally it is clear that $|T|$, $T^{+}$and $T^{-}$are Riesz homomorphisms.

REMARK 5. Let $L$ be a Riesz space with the principal projection property and let $T$ be a disjointness preserving operator from $L$ into an Archimedean Riesz space $M$. If $0 \leqslant u \leqslant e$ in $L$ then there exists a sequence $\left\{f_{n}: n=1,2, \ldots\right\}$ in $L$ such that $f_{n} \rightarrow 0$ (r.u.) and $(|T u|-|T e|)^{+} \leqslant\left|T f_{n}\right|$ for all $n$. Indeed, by the Freudenthal spectral theorem [9, Theorem 40.2] there exists a sequence $\left\{s_{n}: n=1,2, \ldots\right\}$ in $L$ such that $0 \leqslant s_{n} \uparrow u$ (e-uniformly), where each $s_{n}$ is a sum of the form $\sum_{i=1}^{k} \alpha_{i} p_{i}$ with $0 \leqslant \alpha_{i} \leqslant 1(i=1, \ldots, k)$ and $p_{1}, \ldots, p_{k}$ mutually disjoint components of $e$. Using that $T$ is disjointness preserving, it follows easily that $\left|T s_{n}\right| \leqslant|T e|$ and so $(|T u|-|T e|)^{+} \leqslant\left|T\left(u-s_{n}\right)\right|(n=1,2, \ldots)$. Hence we can take $f_{n}=u-s_{n}$. It follows, in particular, that if in this case $T$ has the additional property that $\inf \left|T f_{n}\right|=0$ whenever $f_{n} \rightarrow 0$ (r.u.) in $L$, then $T$ is order bounded (and $0 \leqslant u \leqslant e$ in $L$ implies that $|T u| \leqslant|T e|)$.

As observed by Abramovich [1, §2], if $L$ and $M$ are normed Riesz spaces and $T$ is a norm bounded disjointness preserving operator from $L$ into $M$, then $f_{n}, g_{n} \rightarrow 0$ (r.u.) in $L$ implies that $\inf _{n}\left(\left|T f_{n}\right|+\left|T g_{n}\right|\right)=0$ in $M$, and so $T$ is order bounded. This was also proved in the case when $L$ is a Dedekind $\sigma$-complete Banach lattice by W. Arendt [5, Theorem 2.5].

A linear operator $T$ from an Archimedean Riesz space $L$ into itself is called band preserving if $T(B) \subset B$ for all bands $B$ in $L$ (equivalently, $T f \perp g$ whenever $f \perp g$ in $L$ ). Since a band preserving operator is clearly disjointness preserving, all of the above results apply to band preserving operators. An order bounded band preserving operator is called an orthomorphism. Band preserving operators and orthomorphisms have been studied extensively (see e.g. [2, 6, 7, 8, 12, 13 and 14]). 
We shall present some other applications of Theorem 2. In particular we will prove that under certain conditions on the spaces $L$ and $M$, any disjointness preserving operator from $L$ into $M$ is order bounded on some order dense ideal in $L$.

Let $L$ and $M$ be Archimedean Riesz spaces and let $T$ be a linear operator from $L$ into $M$. If $A$ and $B$ are ideals in $L$ such that the restrictions $T \mid A$ and $T \mid B$ are order bounded, then $T \mid(A+B)$ is order bounded as well. Hence, if $A_{T}$ is the union of all ideals $A$ in $L$ with the property that $T \mid A$ is order bounded, then $A_{T}$ is an ideal in $L$ and $T \mid A_{T}$ is order bounded. Clearly, $A_{T}$ is the largest ideal in $L$ on which $T$ is order bounded. If $T$ is disjointness preserving, then it follows immediately from Corollary 3 that $|T u| \leqslant|T v|$ whenever $0 \leqslant u \leqslant v \in A_{T}$. For band preserving operators we have the following interesting result.

Proposition 6. If $T$ is a band preserving operator on the Archimedean Riesz space $L$, then $A_{T}$ is a band.

Proof. Take $0 \leqslant u \leqslant e \in A_{T}^{d d}$ and suppose that $(|T u|-|T e|)^{+}>0$. Then there exists $v \in A_{T}$ such that $0<v \leqslant(|T u|-|T e|)^{+}$. Let $n$ be a natural number such that $(n v-e)^{+}>0$ and put $p=n v \wedge u$ and $q=n v \wedge e$. Then $0 \leqslant p \leqslant q \in A_{T}$ and so $|T p| \leqslant|T q|$. Since $T$ is band preserving, it follows from $u-p \perp(n v-e)^{+}$and $e-q \perp(n v-e)^{+}$that $T u-T p \perp(n v-e)^{+}$and $T e-T q \perp(n v-e)^{+}$. Now it is easy to see that

$$
(|T u|-|T e|)^{+}-(|T p|-|T q|)^{+} \perp(n v-e)^{+}
$$

and hence $(|T u|-|T e|)^{+} \perp(n v-e)^{+}$. By the choice of $v$ this implies that $(n v-e)^{+}=0$, which is a contradiction. We thus have proved that $|T u| \leqslant|T e|$ whenever $0 \leqslant u \leqslant e \in A_{T}^{d d}$. Therefore $T$ is order bounded on $A_{T}^{d d}$. Now it follows from the definition of $A_{T}$ that $A_{T}=A_{T}^{d d}$; hence $A_{T}$ is a band.

In order to prove the next result we need a lemma.

LemMa 7. Let $L$ and $M$ be Archimedean Riesz spaces and assume that $L$ is uniformly complete. Let $T$ be a disjointness preserving operator from $L$ into $M$. Suppose $0 \leqslant u_{n} \leqslant e_{n} \leqslant e(n=1,2, \ldots)$ in L such that $\left\{e_{n}\right\}_{n=1}^{\infty}$ is a disjoint sequence and put $w_{n}=\left(\left|T u_{n}\right|-\left|T e_{n}\right|\right)^{+}$for all $n$. Then $\left\{w_{n}\right\}_{n=1}^{\infty}$ is a disjoint sequence in $M$ with the property that $\left\{\lambda_{n} w_{n}\right\}_{n=1}^{\infty}$ is order bounded in $M$ for any sequence $\left\{\lambda_{n}\right\}_{n=1}^{\infty}$ of positive real numbers.

Proof. By Theorem 2 there exist for each $n$ two sequences $\left\{f_{n k}\right\}_{k=1}^{\infty}$ and $\left\{g_{n k}\right\}_{k=1}^{\infty}$ in the ideal generated by $e_{n}$, such that $f_{n k}, g_{n k} \rightarrow 0$ (e-uniformly) as $k \rightarrow \infty$ and $0 \leqslant w_{n} \leqslant\left|T f_{n k}\right|+\left|T g_{n k}\right|(k=1,2, \ldots)$ for all $n$. Take positive real numbers $\left\{\lambda_{n}\right\}_{n=1}^{\infty}$. For each $n$ there exists a natural number $k_{n}$ such that

$$
\left|f_{n k_{n}}\right| \leqslant \frac{1}{n^{2} \lambda_{n}} e \text { and }\left|g_{n k_{n}}\right| \leqslant \frac{1}{n^{2} \lambda_{n}} e .
$$

Define

$$
f=\sum_{n=1}^{\infty} \lambda_{n} f_{n k_{n}} \text { and } g=\sum_{n=1}^{\infty} \lambda_{n} g_{n k_{n}}
$$


(e-uniformly convergent series). Since the terms in the series defining $f$ are mutually disjoint and since $T$ is disjointness preserving, it is easy to see that $0 \leqslant \lambda_{n}\left|T f_{n k_{n}}\right| \leqslant|T f|$ for all $n$. Similarly we get that $0 \leqslant \lambda_{n}\left|T g_{n k_{n}}\right| \leqslant|T g|(n=1,2, \ldots)$. Therefore

$$
0 \leqslant \lambda_{n} w_{n} \leqslant \lambda_{n}\left|T f_{n k_{n}}\right|+\lambda_{n}\left|T g_{n k_{n}}\right| \leqslant|T f|+|T g|
$$

for all $n$. Hence $\left\{\lambda_{n} w_{n}\right\}_{n=1}^{\infty}$ is order bounded.

THEOREM 8. Let $L$ and $M$ be Archimedean Riesz spaces such that $L$ is uniformly complete and for each disjoint sequence $0<w_{n} \in M(n=1,2, \ldots)$ there exist positive real numbers $\left\{\lambda_{n}\right\}_{n=1}^{\infty}$ such that $\left\{\lambda_{n} w_{n}\right\}_{n=1}^{\infty}$ is not order bounded in $M$. Then each disjointness preserving operator $T$ from $L$ into $M$ is order bounded on some order dense ideal in $L$.

Proof. Let $A_{T}$ be the largest ideal in $L$ on which $T$ is order bounded. We claim that $A_{T}^{d}=\{0\}$. Indeed, suppose $A_{T}^{d} \neq\{0\}$ and take $0<e \in A_{T}^{d}$. Since the ideal generated by $e$ is not finite dimensional, there exist mutually disjoint elements $0<e_{n} \leqslant e(n=1,2, \ldots)$. Since $T$ is not order bounded on the ideal generated by $e_{n}$, there exists $0 \leqslant u_{n} \leqslant e_{n}$ such that $w_{n}=\left(\left|T u_{n}\right|-\left|T e_{n}\right|\right)^{+}>0(n=1,2, \ldots)$. Now it follows from Lemma 7 that $\left\{\lambda_{n} w_{n}\right\}_{n=1}^{\infty}$ is order bounded for any sequence $\left\{\lambda_{n}\right\}_{n=1}^{\infty}$ of positive real numbers, which contradicts the assumption on $M$. Hence $A_{T}^{d}=\{0\}$, i.e., $A_{T}^{d d}=L$.

The above theorem has the following immediate corollary.

COROllary 9. If $L$ is a Banach lattice and $M$ is a normed Riesz space, then any disjointness preserving operator from $L$ into $M$ is order bounded on some order dense ideal in $L$.

It follows from a combination of Proposition 6 and Theorem 8 that if $L$ is a uniformly complete Riesz space with the property that for each disjoint sequence $0<w_{n} \in L(n=1,2, \ldots)$ there exist positive real numbers $\left\{\lambda_{n}\right\}_{n=1}^{\infty}$ such that $\left\{\lambda_{n} w_{n}\right\}_{n=1}^{\infty}$ is not order bounded, then any band preserving operator in $L$ is an orthomorphism. This implies in particular that any band preserving operator on a Banach lattice is order bounded, and hence norm bounded (as any order bounded operator on a Banach lattice is norm bounded), which is a result of Yu. A. Abramovich, A. I. Veksler and A. V. Koldunov [2, 3]. We note, however, that using Lemma 7 it is not difficult to prove the following result, which is due to A. W. Wickstead (presented at the meeting on Riesz spaces and operator theory in Oberwolfach, 1982): If $L$ is a uniformly complete Riesz space with the property that for each $0<e \in L$ such that the order interval $[0, e]$ does not contain any atoms, there exists a disjoint sequence $\left\{e_{n}\right\}_{n=1}^{\infty}$ in $[0, e]$ such that $\left\{\lambda_{n} e_{n}\right\}_{n=1}^{\infty}$ is not bounded in $L$ for some sequence $\left\{\lambda_{n}\right\}_{n=1}^{\infty}$ of positive real numbers, then each band preserving operator in $L$ is automatically order bounded. The case when $L$ is in addition Dedekind $\sigma$-complete was proved by W. A. J. Luxemburg [8, Theorem 9.9].

The condition that every disjointness preserving operator from an Archimedean Riesz space $L$ into itself is order bounded is quite strong. Indeed, let $P$ be a prime ideal in $L$ which is not uniformly closed. Then there exists a sequence $\left\{f_{n}\right\}_{n=1}^{\infty}$ in $P$ 
and $f_{0} \in L \backslash P$ such that $f_{n} \rightarrow f_{0}$ (r.u.). Let $\varphi$ be any linear functional on $L$ with $\varphi(f)=0$ for all $f \in P$ and $\varphi\left(f_{0}\right)=1$. Take $0<e \in L$ and define the disjointness preserving operator $T$ by $T f=\varphi(f) e$ for all $f \in L$. Since $f_{n} \rightarrow f_{0}$ (r.u.), $T f_{n}=0$ $(n=1,2, \ldots)$ and $T f_{0}=e>0, T$ is not order bounded. Furthemore we note that a proper prime ideal $P$ in $L$ is uniformly closed iff $P$ is a maximal ideal (e.g., this follows from a combination of [9, Theorems 27.1, 33.2 and 60.2]). Therefore, if every disjointness preserving operator in $L$ is order bounded, then every proper prime ideal in $L$ is a maximal ideal. By [9, Theorem 37.6], this last condition is equivalent to the property that the quotient Riesz space $L / A$ is Archimedean for every ideal $A$ in $L$. Riesz spaces with this property are called hyper-Archimedean. We thus have the following result.

THEOREM 10. If $L$ is an Archimedean Riesz space such that any disjointness preserving operator in $L$ is order bounded, then $L$ is hyper-Archimedean.

Finally, we note that if $L$ is uniformly complete and hyper-Archimedean, then every principal ideal in $L$ is finite dimensional [9, Theorem 61.4] and hence any linear operator on $L$ is order bounded.

\section{REFERENCES}

1. Yu. A. Abramovich, Multiplicative representation of the operators preserving disjointness, Indag. Math., Proc. Nederl. Akad. Sci. 45 (1983), 265-279.

2. Yu. A. Abramovich, A. I. Veksler and A. V. Koldunov, On operators preserving disjointness, Soviet Math. Dokl. 20 (1979), 1089-1093.

3. __ Operators preserving disjointness, their continuity and multiplicative representation, Linear Operators and Their Applications, Leningrad, 1981, pp. 13-34. (Russian)

4. C. D. Aliprantis and O. Burkinshaw, Locally solid Riesz spaces, Academic Press, New York, San Francisco and London, 1978.

5. W. Arendt, Spectral properties of Lamperti operators, Indiana Univ. Math. J. 32 (1983), 199-215.

6. S. J. Bernau, Orthomorphisms of archimdean vector lattices, Tech. Rep. 14, Univ. of Texas, Austin, 1979.

7. A. Bigard, K. Keimel and S. Wolfenstein, Groupes et anneaux réticulés, Lecture Notes in Math., vol. 608, Springer-Verlag, Berlin, Heidelberg and New York, 1977.

8. W. A. J. Luxemburg, Some aspects of the theory of Riesz spaces, The University of Arkansas Lecture Notes in Math., Vol. 4, 1979.

9. W. A. J. Luxemburg and A. C. Zaanen, Riesz spaces. I, North-Holland, Amsterdam and London, 1971.

10. M. Meyer, Le stabilisatuer d'un espace vectoriel réticulé, C.R. Acad. Sci. Paris Ser. A 283 (1976), $249-250$.

11. Quelques propriétés des homomorphismes d'espaces vectoriels réticulés, Equipe d'Analyse, E.R.A. 294, Université de Paris VI, 1979.

12. B. de Pagter, f-algebras and orthomorphisms, Thesis, University of Leiden, 1981.

13. A. W. Wickstead, Extensions of orthomorphisms, J. Austral. Math. Soc. Ser. A 29 (1980), 87-98.

14. A. C. Zaanen, Examples of orthomorphisms, J. Approx. Theory 13 (1975), 192-204.

Department of Mathematics, California institute of Technology, Pasadena, California 91125

Current address: Department of Mathematics, Delft University of Technology, Delft, The Netherlands 